\title{
Fatores de incorporação de requisitos ambientais no processo de desenvolvimento de produtos em indústrias de móveis sob encomenda
}

\author{
Incorporation of environmental requirements in the process of developing products in industries \\ from custom furniture
}

Patrícia Silva de Azevedo ${ }^{\mathrm{I}}$ Adriana Maria Nolasco ${ }^{\mathrm{II}}$

\section{RESUMO}

Este estudo teve por objetivo identificar os requisitos ambientais incorporados em arranjos produtivos locais de móveis e os fatores internos e externos para a aplicação desses requisitos no processo de desenvolvimento de produtos. Foram analisadas 18 micro e pequenas empresas do Pólo Moveleiro de Itatiba, São Paulo (SP), quanto à adoção de requisitos ambientais no ciclo de vida do produto, assim como no PDP (Processo de Desenvolvimento de Produtos) sob encomenda. Os dados foram coletados por meio de entrevistas semi-estruturadas e visitas in loco, analisando cada atividade do PDP e os requisitos ambientais associados a elas e identificando os responsáveis por cada atividade e, ao final, os fatores que motivaram a incorporação desses requisitos. Os resultados indicam que os fatores econômicos ainda ditam a forma das empresas responderem às questões ambientais, seja a adequação às leis e aos regulamentos ou a redução dos custos de produção. Além disso, a ausência de profissionais capacitados na área de desenvolvimento de produtos sustentáveis propicia o aumento das dificuldades em estruturar o setor.

Palavras-chave: ciclo de vida, processo produtivo, indústria moveleira.

\section{ABSTRACT}

This research had the goal of identifying the environmental requirements incorporated in furniture clusters and the internal and external factors for the use of those requirements in the product development process. Eighteen micro and small companies from the furniture hub of Itatiba (a city in the Brazilian state of São Paulo) were analyzed according to their adopting environmental requirements in the products life cycle as well as in the PDP (product development process) of custom orders. The data were collected through partly open interviews and on site visitations, by analyzing each activity in the PDP and the environmental requirements linked to them, identifying the people responsible for each activity and, in the end, identifying the factors that motivated the adoption of those requirements. The results show that economic factors still rule the way in which the companies respond to environmental issues, neither by adaption to the laws and regulations or by reducing the production costs. Moreover, the lack of capable professionals in the area of developing sustainable products contributes for making it more difficult to structure the sector.

Key words: life cycle, productive process, furniture industry.

\section{INTRODUÇÃO}

O desenvolvimento de produtos no contexto florestal é um processo complexo, transformando dados sobre oportunidades de mercado e requisitos estéticos, normativos, funcionais e operacionais em informações para a fabricação de um produto comercial (CLARK \& FUJIMOTO, 1991; WHEELWRIGHT \& CLARK, 1992).

A partir da década de 1980, iniciou-se a incorporação dos requisitos ambientais ao processo de desenvolvimento de produtos (PDP), por meio do estudo do seu ciclo de vida (Life Cicle Assessment), em resposta a crescentes pressões sociais para a inserção da gestão ambiental no âmbito de planejamento estratégico do setor industrial, rumo a uma maior sustentabilidade nos sistemas de produção (VEZZOLI, 2007).

'Escola Superior de Agricultura "Luiz de Queiroz” (ESALQ), Universidade de São Paulo (USP), 13418-900, Piracicaba, SP, Brasil. E-mail: psazeved@hotmail.com. Autor para correspondência.

"Departamento de Ciências Florestais, ESALQ, USP, Piracicaba, SP, Brasil. 
A etapa de planejamento de projetos na indústria moveleira é uma das mais importantes para reduzir os impactos negativos da produção. Nesse caso, a escolha por requisitos ambientais para o desenvolvimento de móveis deve atender não apenas aspectos processuais, mas também sócio-culturais.

Na cadeia produtiva de móveis em madeira, COSTA \& GOUVINHAS (2003) mencionam os pontos ambientais significativos, tais como as condições ambientais locais e regionais para produção e distribuição do produto, as características dos seus produtos, os requisitos legais e de mercado e a disponibilidade de recursos humanos, financeiros e materiais.

No Brasil, o setor moveleiro é formado por mais de 16.000 micros, pequenas e médias empresas, gerando cerca de 190.000 empregos. São, em geral, empresas familiares, de capital nacional, fortemente fragmentadas e verticalizadas. Localizam-se, em sua maioria, nas regiões Sul e Sudeste e produzem móveis residenciais e de escritório. Quanto às matérias-primas, predomina o uso de serrados de florestas nativas e plantadas, compensados e painéis reconstituídos (ABIMÓVEL, 2006).

A produção sob encomenda é o modelo produtivo predominante no setor, especialmente nas micro e pequenas empresas. Segundo RUSSOMANO (1979), esse processo produtivo se caracteriza por produzir grande variedade de artigos em pequena quantidade e por demandar maior tempo no desenvolvimento dos produtos e no planejamento da produção em relação ao tempo de fabricação.

Dessa forma, as indústrias de móveis sob encomenda são as mais atingidas pelas dificuldades em adaptar os seus processos industriais e de desenvolvimento de produtos a sua cultura empresarial, ao seu modelo gerencial e aos novos desafios de conformidade ambiental e do desenvolvimento sustentável. Além disso, ela está inserida no setor de base florestal, que, por suas características, é foco de fortes apelos ambientais, resultado do desenvolvimento de políticas públicas voltadas à conservação dos recursos naturais.

O objetivo do trabalho foi identificar os fatores motivadores da inclusão dos requisitos ambientais no processo de desenvolvimento de móveis em micro e pequenas empresas com produção sob encomenda.

\section{MATERIAL E MÉTODOS}

Utilizou-se o método de estudo de caso, ou investigação empírica, no Município de Itatiba, São
Paulo (SP), considerado na década de 1970 a "Capital do Móvel Colonial”, utilizando madeira maciça comumente retirada de florestas nativas no Norte e Nordeste do país.

As empresas foram selecionadas por meio de uma amostragem intencional, não probabilística, de forma a privilegiar a coleta qualitativa das informações. Para tanto, foram utilizados dados do Sindicato da Indústria Moveleira de Itatiba (SIDIMOVEIS) e do cadastro do programa "Móveis Itatiba", realizado no ano de 2004, pelo SENAI "Luiz Scavone", havendo apenas 18 empresas que se disponibilizaram a participar da pesquisa.

Foram identificadas as fases do PDP no processo sob encomenda, conforme PIRES (1995), considerando os principais responsáveis nas tomadas de decisão nos três níveis de planejamento (estratégico - diretor/proprietário; projetual - designer/projetista; e produtivo - gerente de produção/encarregado), as atividades de cada fase e os sistemas de controle, segundo modelo de referência para desenvolvimento de produtos (AMARAL et al., 2006), e quais os requisitos ambientais considerados no ciclo de vida do produto, relacionando-os aos conceitos citados por MANZINI \& VEZZOLI(2005).

Para identificar os requisitos ambientais pelas empresas moveleiras, primeiramente utilizou-se a escala Likert (ALBAUM, 1997), adaptada aos parâmetros da pesquisa, para avaliar o nível de conhecimento sobre o tema. Essa escala variou de "não conhece e não aplica requisitos ambientais”, com valor numérico 1; "conhece conceitualmente e não aplica", com valor numérico 2; "conhece conceitualmente e aplica de forma parcial”, com valor numérico 3; "conhece conceitualmente e aplica de forma completa", com valor numérico 4 e "implementa práticas ambientais inovadoras e cria de modelos" no processo de desenvolvimento de produtos.

Para gerar valores ponderados de frequência para cada requisito aplicado, realizou-se o produto das frequências absolutas pelo valor dos critérios aplicados, de acordo com a expressão matemática:

$\mathrm{VP}=f_{i} \times v_{i}$, sendo o valor ponderado $(\mathrm{VP})$ igual à multiplicação entre a frequência em cada resposta dada $\left(f_{i}\right)$ e o valor associado a elas $\left(v_{i}\right)$.

Posteriormente, foram identificados os requisitos aplicados pelas empresas correspondentes aos valores ponderados obtidos e a nomenclatura por ela utilizada.

Após a obtenção do valor ponderado, verificaram-se as etapas do ciclo de vida dos produtos, 
listando os principais requisitos ambientais relacionados às atividades do PDP.

Com a identificação desses requisitos ambientais, analisaram-se os fatores de motivação das empresas estudadas para a aplicação dessas ações, identificando quais setores e quem gerenciava as decisões dentro da rotina de trabalho das empresas, caracterizando o processo de incorporação de requisitos ambientais no desenvolvimento de móveis sob encomenda.

\section{RESULTADOS E DISCUSSÃO}

A figura 1 apresenta o fluxograma da produção sob encomenda nas micro e pequenas indústrias de móveis. O fluxo de produção representa as etapas do processo, que, por se tratar de produção sob encomenda, as necessidades dos clientes devem ser traduzidas em requisitos do projeto.

A primeira etapa no processo de desenvolvimento de produtos é a elaboração de um

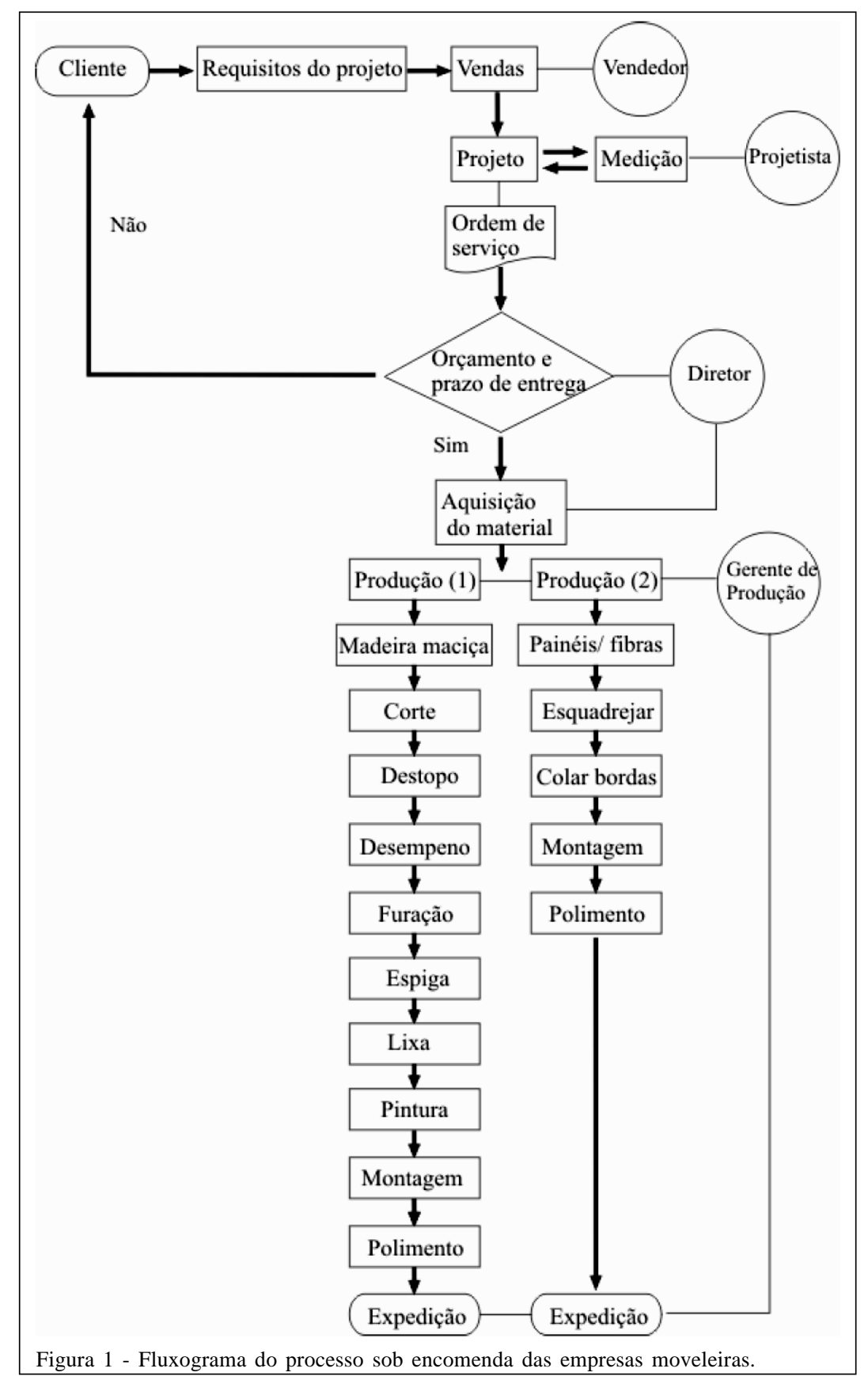

Ciência Rural, v.39, n.8, nov, 2009. 
pré-projeto que será aprovado pelo cliente, decorrendo das especificações dos requisitos projetuais, que devem estar atrelados aos ambientais, sendo estes detalhados e formalizados para a execução do produto final.

As relações entre o processo sob encomenda, com cada etapa do PDP, e os requisitos ambientais inseridos no ciclo de vida do produto são apresentadas na tabela 1. Essa relação apresenta a afinidade entre cada etapa de desenvolvimento, possibilitando a identificação dos requisitos ambientais por atividade.

A partir desses resultados, pôde-se identificar que esses requisitos ainda são pouco incorporados nas atividades de desenvolvimento de produtos ou mesmo nas decisões do processo de fabricação, decorrente da falta de conhecimento sobre gerenciamento e sustentabilidade (Tabela 2).

Os valores ponderados dos requisitos ambientais mencionados pelas empresas e relacionados ao ciclo de vida nas macrofases do PDP sob encomenda são apresentados na figura 2. Observa-se que as principais práticas de incorporação dos requisitos ambientais se encontram nas fases do PDP de préprojeto, produção e entrega, nas etapas de ciclo de vida, na pré-produção, na produção, no uso e na distribuição.
Com o valor ponderado de 72 , o aproveitamento de peças e componentes na fase de produção foi o requisito que apresentou maior representatividade, seguido da utilização de material renovável (64), definido como alternativas de novos materiais

As alternativas de redesign, considerado pelas empresas como reúso de sistemas, subsistemas e componentes (24), utiliza o aproveitando dos processos de desenvolvimento de projetos posteriores para buscar as melhores soluções em projetos atuais.

Nesse setor, é comum, nas pequenas empresas, o uso de programas de computador com a modulação de móveis ou projetos prontos, com o registro dos trabalhos anteriores, ocorrendo da mesma forma para o planejamento de distribuição (24) no setor de expedição e transporte.

O valor ponderado de 18 relaciona-se ao prolongamento da vida do produto, considerado pelo consumidor um forte atributo para bens duráveis, como móveis.

Diante dos requisitos ambientais encontrados, relacionaram-se na tabela 3 os fatores motivadores para a sua incorporação no processo de desenvolvimento de produtos.

Tabela 1 - Fases do Processo de Desenvolvimento de Produtos responsáveis pelas atividades e pelos requisitos ambientais.

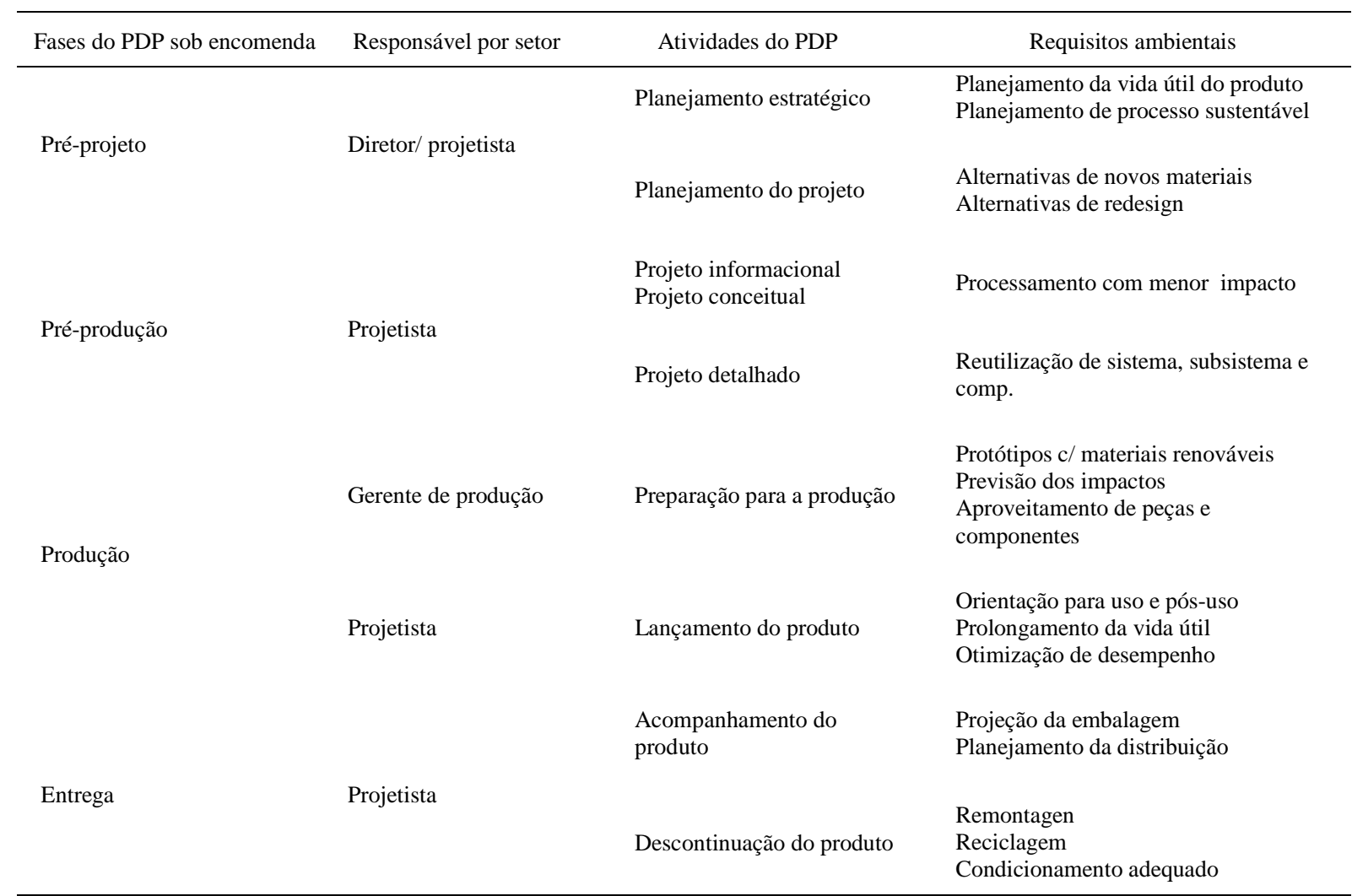


Tabela 2 - Requisitos ambientais considerados pelas empresas.

\begin{tabular}{lcc}
\hline Requisitos ambientais considerados pelas empresas & Frequência relativa (\%) & Valor ponderado \\
\hline Reúso de peças e componentes & 100 & 72 \\
Aquisição de matéria-prima renovável & 89 & 64 \\
Reúso de sistemas, subsistemas e componentes & 44 & 24 \\
Planejamento de distribuição (logística) & 44 & 24 \\
Interação com mercado exigente & 33 & 18 \\
Prolongamento da vida útil & 33 & 18 \\
Marketing ecológico & 22 & 12 \\
\hline
\end{tabular}

Em 10 empresas o fator interno de redução de custos no projeto e processo foi o mais mencionado, relacionando-se a percepção pela direção da empresa da falta de planejamento e controle das atividades, e aplicando requisitos de reúso de sistemas e componentes para minimizar desperdícios.

Seis empresas apresentaram como fatores as exigências do mercado consumidor, que principalmente, por requisitos de durabilidade do produto, agregam valores ambientais e de qualidade da matéria-prima como exigências na produção dos móveis.

Ainda como fator externo à empresa, a adequação a leis e resoluções ambientais são razões de uso de requisitos ambientais relacionados à matériaprima, devendo esta ser certificada ou apresentar

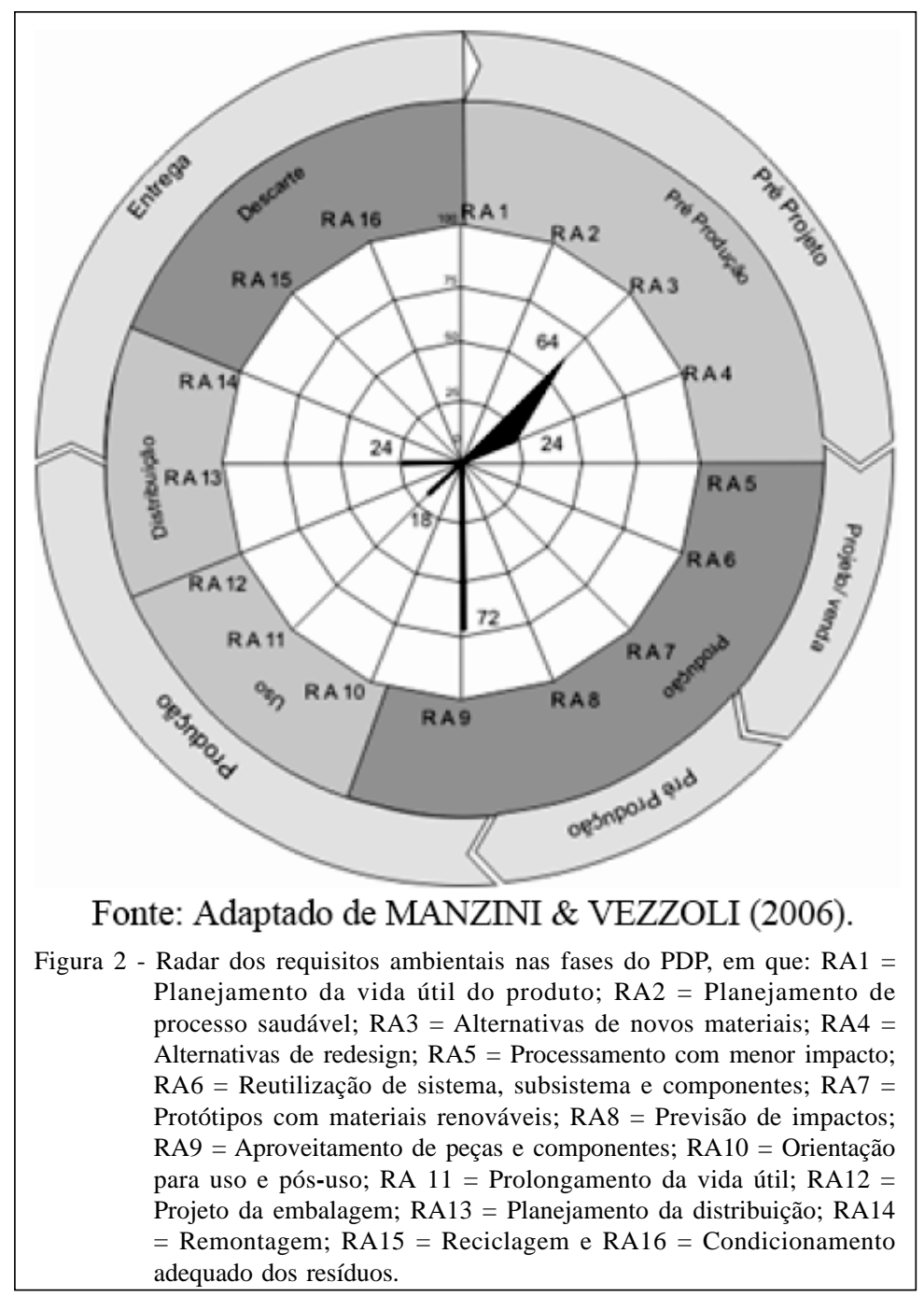

Ciência Rural, v.39, n.8, nov, 2009. 
Tabela 3 - Fatores motivadores e requisitos ambientais aplicados nas empresas.

\begin{tabular}{lcc}
\hline Fatores motivadores para incorporação de requisitos ambientais & Tipo de fator & No de empresas \\
\hline Redução de custos no projeto e processo & Interno & 10 \\
Exigências do mercado consumidor & Externo & 6 \\
Legislação e resoluções ambientais & Externo & 4 \\
Aumento da competitividade & Externo & 2 \\
\hline
\end{tabular}

registro de extração de áreas manejadas e autorização para transporte.

O último fator mencionado é o de viabilizar o aumento da competitividade entre as empresas do pólo moveleiro, utilizando-se meios de diferenciação como o marketing ecológico, que atualmente é visto por muitos empresários como modismo. Contudo, são perceptíveis os benefícios do uso de requisitos ambientais no PDP, não apenas pela tendência mercadológica, mas também como forma de otimizar a produção, diminuir gastos, racionalizar o uso de materiais e ferramentas e, principalmente, demonstrar respeito pelo capital natural gerador de energia e insumos.

Observou-se que as práticas ambientais consideradas por todas as empresas do pólo de Itatiba demonstram preocupação econômica do sistema produtivo, devido ao fato de estarem ligadas a custos, com adequações às legislações ou exigências comerciais. Essa atitude reativa dificulta o investimento em tecnologias "limpas", ou mesmo, a responsabilidade sobre o desenvolvimento dos seus produtos.

Essa realidade exprime o quando as micro e pequenas empresas moveleiras com sistema sob encomenda ainda apresentam um foco exclusivo em atender as exigências dos requisitos projetuais do consumidor, que nem sempre demonstra conhecimento sobre processos sustentáveis ou opta por soluções inovadoras e com menor impacto ambiental. Assim, cabe à empresa uma melhor estruturação de seu sistema produtivo e do uso de estratégias para planejar ações proativas.

\section{CONCLUSÕES}

O processo de inserção dos requisitos ambientais em micro e pequenas empresas é caracterizado por fatores internos e externos, relacionados principalmente aos aspectos econômicos na tomada de decisão. Minimização de gastos no processo e nos materiais utilizados e adequação às leis e regulamentações são os principais motivadores, fazendo com que as empresas se direcionem somente a ações corretivas no PDP.
O modelo de gestão ambiental predominante nas indústrias de móveis sob encomenda no Pólo Moveleiro de Itatiba - SP é quase inexistente, baseado na adoção tímida de requisitos ambientais, sendo esses identificados principalmente na etapa de descarte, caracterizando ações corretivas do processo.

\section{REFERÊNCIAS}

ASSOCIAÇÃO BRASILEIRA DAS INDÚSTRIAS DO MOBILIÁRIO - ABIMÓVEL. Panorama do setor moveleiro no Brasil: informações gerais - dez. 2006. Disponível em: <http://www.sebraego.com.br/site/arquivos/downloads/ Panorama_do_Setor_Moveleiro_no_Brasil_23758.pdf $>$. Acesso em: 20 fev. 2008.

ALBAUM, G. The Likert Scale Revisited. Journal of the Market Research Society, Birmingham, v.39, n.2, p.33148, 1997.

AMARAL, D.C. et al. Gestão do desenvolvimento de produtos: uma referência para a melhoria do processo. São Paulo: Saraiva, 2006. 542p.

CLARK, K.B.; FUJIMOTO, T. Product development performance: strategy, organization and management in the world auto industry. Boston: Harvard Business School, 1991. 234p.

COSTA, G.J.; GOUVINHAS, R.P. As estratégias de ecodesign e o processo de desenvolvimento de produto em pequenas e médias empresas do nordeste e sudeste do Brasil: um estudo comparativo. In: CONGRESSO BRASILEIRO DE GESTÃO DE DESENVOLVIMENTO DE PRODUTO, 4., 2003, Gramado. Anais... Gramado: CBGDP, 2003. CD ROM.

MANZINI. E.; VEZOLLI, C. O desenvolvimento de produtos sustentáveis: os requisitos ambientais dos produtos industriais. São Paulo: Edusp, 2005. 368p.

PIRES, S.R.I. Gestão estratégica da produção. Piracicaba: UNIMEP, 1995. 269p.

RUSSOMANO, V.H. Planejamento e acompanhamento da produção. São Paulo: Pioneira, 1979. Cap.3, p.37-47.

VEZZOLI, C. System design for sustainability: theory, methodsand tools for a sustainable "satisfactionsystem”design. Milan: Maggioli editore, 2007. 260p.

WHEELWRIGHT, S.C.; CLARK, K.B. Revolutionizing product development: quantum leaps in speed, efficiency, and quality. New York: The Free, 1992. 369p. 